\title{
Use of WIMS/PANTHER to Analyse Nitrogen Worth Measurements at the UK Fleet of AGRs
}

\author{
Eddie Birkett, Martin Knight
}

\author{
EDF Energy, Barnett Way, Barnwood, Gloucester, GL4 3RS, UK \\ eddie.birkett@edf-energy.com
}

\begin{abstract}
The UK fleet of Advanced Gas-Cooled Reactors (AGRs) are moderated with graphite and cooled with carbon dioxide. Each reactor consists of over 300 fuel channels with a cluster of UO2 fuel pins surrounded by graphite. Primary shutdown is achieved with control rods, which are at interstitial locations at six AGR stations, and in vacated fuel channels in the seventh. Nitrogen injection into the coolant is used for diverse shutdown or hold down.

The reactivity worth of nitrogen was measured during cold commissioning at each AGR during the 1970s and 1980s. This measurement involved inducing a flux transient through a known change in nitrogen pressure, with conversion into a reactivity worth using kinetic data. Noting the time elapsed since this work (over 40 years) it is sensible to review this validation and reconsider these measurements using a consistent source of kinetic data.

The lattice code WIMS and the reactor code PANTHER can be used to analyse the commissioning cores, in particular to determine the core-wide kinetic data. Furthermore the calculated worth of nitrogen can be compared with measurement. For the six interstitial AGR reactor designs nitrogen worth is under-predicted by about $10 \%$ and by about $5 \%$ for the inchannel design.

This consistent under-prediction is investigated further with SERPENT.
\end{abstract}

KEYWORDS: Nitrogen, AGR, Graphite, Commissioning, Measurement

\section{INTRODUCTION}

This paper discusses the use of station commissioning measurements as a source of validation for modern methods, codes and data.

Advanced gas-cooled reactor (AGR) fuel is a lattice of UO2 pins clad with stainless steel; moderation is provided by graphite and cooling by carbon dioxide. If injected, nitrogen mixes with the coolant until uniformly distributed. Using WIMS/PANTHER the equilibrium state can be modelled and a respective nitrogen worth derived which can be compared with original values.

Nitrogen injection is used across the fleet of AGR reactors as a means of diverse shutdown and/or hold down; though only the latter is considered in this paper. Station commissioning included 
nitrogen or air injection tests which were used to derive the effective worth of nitrogen in the core for the AGR fleet (using point kinetics).

\section{SUMMARY OF CODES AND DATA USAGE}

Calculations use the standard "two step" approach. WIMS and PANTHER are used as the lattice and whole core physics codes respectively. Code-to-code comparisons are made against the Monte Carlo code SERPENT. Details of the code and data versions are provided:

- WIMS: Code version 10A RU1 and a JEF2.2-based nuclear data library [1]. Two solution methods are used:

- PIJ: Collision probabilities, also the method used to derive PANTHER nuclear data.

- CACTUS: Method of characteristics, reference solution in WIMS.

- PANTHER: Code version 5.6 and a WIMS-based nuclear data library [2] (WNDL, derived with WIMS 9A RU1 using JEF2.2 data [3]).

- SERPENT: Code version 2.1.29 and a JEFF3.3-based nuclear data library [4].

\section{STATION COMMISSIONING MEASUREMENTS}

During initial AGR commissioning, all stations assessed the worth of nitrogen via pressure tests. This was either conducted in air (with nitrogen the only significant absorber) or in nitrogen itself. In either case, the pressure change ensured that the test measured the full worth of nitrogen in voids and graphite pores, uniformly across the core.

The graphite moderator is porous and in normal operation these pores contain carbon dioxide. Injected nitrogen will also enter these open volumes, either forcefully (in the presence of a pressure differential, $\mathrm{dP}$ ) or by gaseous diffusion (when $\mathrm{dP} \simeq 0$ ). The pore penetration rate is essentially immediate if driven by a change in pressure, but very much slower, taking many hours/days, if driven by diffusion. Determining the exact time taken is crucial to assessments of the performance of nitrogen injection as a diverse hold down system.

\subsection{Measured Air/Nitrogen Pressure Coefficients}

Early tests were monitored as flux 'divergences', and lasted long enough for stable doubling/halving rates to be seen following a pressure change. This was converted into a reactivity using the 'Inhour' equation (1) with a set of kinetic data, most particularly delayed neutron data. An increase of $5 \%$ in delayed neutron fraction results in about a 5\% increase in the 'measured' worth.

$$
\rho_{s}=-\alpha \Lambda-\sum_{i=1}^{6} \frac{\alpha \beta_{i}}{-\alpha+\lambda^{i}}
$$




\begin{tabular}{l||l|l||l|l||r} 
Stn & $\begin{array}{l}\text { Original } \\
\beta_{\text {eff }}\end{array}$ & $\begin{array}{l}\text { WNDL } \\
\beta_{e f f}\end{array}$ & $\begin{array}{l}\text { Original } \\
\text { Meas }(\mathrm{pcm})\end{array}$ & $\begin{array}{l}\text { Adjusted } \\
\text { Meas }(\mathrm{pcm})\end{array}$ & $\begin{array}{r}\text { PANTHER } \\
\text { Diff }(\%)\end{array}$ \\
\hline $\mathrm{A}$ & $6.930 \%$ & $7.231 \%$ & $497 / \mathrm{atmA}$ & $519 / \mathrm{atmA}$ & -6.2 \\
$\mathrm{~B}$ & $6.930 \%$ & $7.211 \%$ & $538 / \mathrm{bar}$ & $560 / \mathrm{bar}$ & -9.4 \\
$\mathrm{C}$ & $7.285 \%$ & $7.211 \%$ & $710 / \mathrm{bar}$ & $703 / \mathrm{bar}$ & -11.4 \\
$\mathrm{D}$ & $7.184 \%$ & $7.191 \%$ & $778 / \mathrm{barN}$ & $779 / \mathrm{barN}$ & -13.8 \\
$\mathrm{E}$ & $7.184 \%$ & $7.190 \%$ & $755 / \mathrm{barN}$ & $756 / \mathrm{barN}$ & -11.1
\end{tabular}

Table I: Pressure Coefficient Measurements Analysed with WNDL Kinetic Data.

Later tests used 'inverse kinetics' to deduce reactivity during a continuous nitrogen pressure change. This also converted flux changes to reactivity using kinetic data, with the same sensitivity to delayed neutron fraction.

At some stations both approaches were used, with the conclusion that they were broadly equivalent.

Over the period of AGR commissioning, 1975 to 1988, the accepted source of kinetic data was changed quite significantly. To be as consistent as possible, results presented here are 'measured' nitrogen worths as if derived using the kinetic data from the WIMS nuclear data library. The AGR delayed neutron importance has been taken to be 0.99 , and so the delayed data has also been scaled by this factor.

Table I shows variations across the fleet in consistently derived effective delayed fractions are less than $1 \%$; this is reduced from $5 \%$ for different sources of delayed data. This justifies the need to use consistent data for all measurements.

\subsection{PANTHER Analysis of Pressure Coefficient Tests}

The purpose of these tests was to measure the reactivity impact of a change in air or nitrogen pressure. This provides a direct assessment of the worth of a uniform nitrogen distribution at all stations, albeit at cold commissioning.

The tests were carried out at 'room temperature', and at multiple rod configurations however results are only presented for all rods out (ARO). Calculations have been setup to model these conditions.

For a PANTHER simulation of the test, the initial core conditions are used with fresh fuel and zero xenon concentration. This state has broadly the right core loading, except for a range of in-core monitoring devices (absorbers) mostly loaded in peripheral, vacated fuel channels.

Nitrogen is modelled in PANTHER calculations by interpolating to the effective partial pressure in both coolant and moderator; i.e. modelled with full penetration into the graphite pores. The worth of nitrogen is roughly $40 \%$ higher once full penetration is achieved compared to uniform dispersion in the coolant only. 


\subsection{Comparison of Prediction and Measurement}

The aim of these comparisons is to provide as consistent a picture as possible across all stations. To this end, a common source of kinetic data (WNDL) is used to interpret the measurement, and a common nuclear data library (WNDL) is used in PANTHER with suitable corrections to reproduce the true nitrogen content in graphite.

Excluding Station A which has a fundamentally different channel layout, and allowing for known empty channels at Station D, the range of comparisons with measurement is $-9 \%$ to $-12 \%$, see Table I. The range of under-predictions is similar to previous internal analysis undertaken at the time which was based on different modelling performed with different codes.

Note that in no case is there an over-prediction of nitrogen worth, even though the nitrogen content is modelled with full penetration of nitrogen into the pores.

\section{ASSESSMENT OF CURRENT NITROGEN WORTH}

On the basis of these analyses of the commissioning tests, it is judged that the current PANTHER model, based on WNDLs, is suitable for predicting nitrogen worths. It is proposed that this still holds for a current core, taking due account of rod positions and temperatures along with full diffusion of nitrogen into the pores.

Core conditions have changed quite significantly since commissioning tests were undertaken at station. In order to extend validation from the inital plant conditions to current operating conditions and provide additional justification to the judgement, above code-to-code comparisons are made between single assembly calculations with WIMS and the Monte-Carlo code SERPENT.

A calculation is done with and without uniformly distributed nitrogen in the coolant only to derive respective worths. Three cases have been considered:

- TEST: Representative of station commissioning pressure tests with low enrichment, unirradiated fuel in cold conditions.

- SOL: Updated with current design of fuel (including increased enrichment) at zero burnup and hot operating conditions (start of life).

- MOL: Isotopics in the fuel are revised to represent the change from uranium-biased to plutoniumbiased fission expected as a result of irradiation.

Table II shows very good agreement between SERPENT and WIMS calculations, with the WIMS nitrogen worth about 1-2\% less than Monte Carlo using PIJ and 0-1\% with CACTUS. Importantly, WIMS-CACTUS and SERPENT results improve agreement with commissioning measurements. This provides evidence that if an alternate set of lattice data were to be used by PANTHER, a small improvement to the level of accuracy could be achieved.

Additionally, it demonstrates improved agreement at the code-to-code level for current plant and fuel conditions. 


\begin{tabular}{l||l|l|l||r|r} 
Case & $\begin{array}{l}\text { WIMS-PIJ } \\
(\mathrm{pcm})\end{array}$ & $\begin{array}{l}\text { WIMS-CACTUS } \\
(\mathrm{pcm})\end{array}$ & $\begin{array}{l}\text { SERPENT } \\
(\mathrm{pcm})\end{array}$ & $\begin{array}{r}\text { Diff WP-S } \\
(\%)\end{array}$ & $\begin{array}{r}\text { Diff WC-S } \\
(\%)\end{array}$ \\
\hline TEST & 2128 & 2146 & 2167 & -1.8 & -1.0 \\
SOL & 1415 & 1427 & 1432 & -1.2 & -0.3 \\
MOL & 1366 & 1380 & 1380 & -1.0 & 0.0
\end{tabular}

Table II: Nitrogen Worths Comparisons with WIMS and SERPENT. All SERPENT results are converged to within 5 pcm.

\section{DISCUSSION OF OTHER CONSIDERATIONS}

\subsection{Graphite Weight Loss}

When nitrogen is injected into the circuit, the coolant gas becomes a mixture of $\mathrm{CO} 2$ and N2. Depending on the level of pressure control there may be a variation in total pressure or not. In the absence of any pressure variation, N2 will only enter graphite pores via diffusion, which is a slow process.

The rate of diffusion into the pores is governed by a number of factors:

- the graphite brick geometry and gas access via holes

- the diffusivity of the initial graphite

- the impact of Graphite Weight Loss (GWL)

The first two of these are fixed prior to commissioning meaning that only GWL will result in changes to penetration rate into the pores; it also affects the size of pores (which logically follows due to the loss of the graphite from the moderator).

The core has been modelled with zero GWL throughout presented analysis. In real terms its effect would be to increase the worth of nitrogen and reduce the time taken to achieve uniform distribution thereby further increasing the reactivity benefit it provides for hold down.

\subsection{8-group Kinetic Data}

Although consistency was the primary intent of comparisons with station commissioning tests, which has been achieved with WNDLs across the fleet, it is noted that kinetic data has moved on from the values used as part of this analysis. In particular, the use of 8-group kinetic data is now prevalent in modern nuclear data libraries, such as JEFF3.3.

Sensitivity analysis has been undertaken to derive the impact of the modern 8-group kinetic data approach. Using the same consistent approach across the AGR fleet, re-derived measured worths were calculated to be up to $1 \%$ lower. Again this reduces the observed under-prediction, but it is a small contribution. 
Calculations were done using WIMS (JEFF3.3 data) and SERPENT (Meulekamp kinetic data, noting this is actually $\beta_{\text {eff }}$ ).

\section{CONCLUSIONS}

Station commissioning pressure tests from the 1970s/1980s have been used as a proposed source of validation for modern codes, data and methods.

Measured worths were re-derived using consistent kinetic data and used as the basis of comparisons with a WIMS/PANTHER whole core calculation. Analysis of the pressure tests at commissioning conditions highlighted a general under-prediction of $-9 \%$ to $-12 \%$ against the revised measured worths.

Code-to-code comparisons were used to investigate sensitivities to these analyses. The use of either WIMS-CACTUS or the Monte Carlo code SERPENT to derive lattice nitrogen worths and to supply kinetic data to derive 'measurements' would both offer small improvements, reducing the discrepancy against measurement by about $2-3 \%$.

\section{REFERENCES}

[1] B. A. Lindley, T. D. Newton, J. G. Hosking, P. N. Smith, D. J. Powney, B. Tollit, and P. J. Smith. "Release of WIMS10: A Versatile Reactor Physics Code for Thermal and Fast Systems." In Proceedings of ICAPP 2015, Nice, France (May 2015).

[2] P. K. Hutt, N. Gaines, M. McEllin, R. J. White, and M. J. Halsall. "The UK Core Performance Code Package.” Nuclear Energy, volume 30, No.5, pp. 291-298 (1991).

[3] T. D. Newton and J. L. Hutton. "The Next Generation Lattice Code: WIMS9." In International Conference on the Physics of Reactors, PHYSOR 2002, Seoul, Korea (October 2002).

[4] J. Leppänen, M. Pusa, T. Viitanen, V. Valtavirta, and T. Kaltiaisenaho. "The Serpent Monte Carlo Code: Status, Development and Applications in 2013." Annals of Nuclear Energy, volume 82, pp. 142-150 (2015). 